\title{
DESIGN ANALYSIS OF A LOW-COST PARABOLIC ANTENNA FOR DEVELOPING NATION
}

\author{
ONAIFO FRANK ${ }^{* 1}$, OKANDEJI ALEXANDER AKPOFURE ${ }^{1}$, IDOWU FISAYO ${ }^{1}$, \\ OLASUNKANMI OMOWUMMI GRACE ${ }^{1}$, OLALUWOYE OLAWALE ${ }^{2}$, \\ FOLORUNSHO OLAOLU ${ }^{2}$
}

\author{
${ }^{1}$ Department of Electrical/Electronic Engineering, Olabisi Onabanjo University, Ogun \\ State, Nigeria \\ ${ }^{2}$ Department of Computer Engineering, Olabisi Onabanjo University, Ogun State, Nigeria
}

\begin{abstract}
This paper is aimed at designing a locally fabricated fiberglass parabolic antenna as a replacement for a metallic fabricated parabolic antenna. They are coated with a thin film of aluminum dust to enhance reflectivity since electromagnetic radiations do not travel deep in a conductor. This reduces the thick mass of metal usually used for constructing metallic parabolic antenna leading to a reduced cost of production. It incorporates a Low Noise Block (LNB) Converter comprising of amplifiers and frequency down converter. Metallic parabolic antenna contributes more to signal loss by reflecting radio waves. The Parabolic antenna is designed to receive microwave signals in the K-band with the down converter converting it to very high frequency (VHF) and ultra-high frequency (UHF) employing the super-heterodyning principle. The study shows the design and installation of a low-cost parabolic antenna in the rural community in Nigeria. The designed parabolic antenna is used to receive a signal with the center frequency of $11.3 \mathrm{GHz}$ and signal strength of $60 \%$. The economic viability is that fiber-glass Parabolic antenna can replace the metallic fabricated ones.
\end{abstract}

Keywords: Fiber-glass parabolic antenna, Metallic parabolic antenna, Reflectivity, Signal strength, Production cost

\section{INTRODUCTION}

Antennas have played and will continue to play a great role in communication for transmission and reception of radio signals. A cheaper antenna that meets the need of developing nation is explored through the development of a fiberglass parabolic antenna. The cost of importing parabolic dish antenna into a developing nation like Nigeria is very prohibitive and as the nation migrates to digital broadcasting, an alternative means of massproducing parabolic dish antenna is inevitable. This paper tends to address this [1].

According to Morozov et al., (2018) parabolic antenna has high directivity with a narrow beam width. This has placed it more advantageous with higher gain compared to other forms of antennas. The use of antennas has spread from communication purposes to space exploration [2]. Janhunen et al., 2018 investigate the possibility of using an antenna to explore other galaxies. The possibility of using a parabolic reflector in their paper is quite promising [3]. Their work is quite ambitious by proposing to build a $1 \mathrm{~km}$ diameter parabolic antenna. Ullah et al., 2015 presented a low-cost microstrip planar antenna for use in a communication system [4]. Their work

\footnotetext{
* Corresponding author, email: excitefranko@yahoo.com

(C) 2020 Alma Mater Publishing House
} 
suffers from the fact that their antenna is low power and cannot transmit over a large distance. Papageorgiou, 2012 discusses the use of parabolic reflectors and others at the upper end of the microwave region with a higher bandwidth and data rate [5].

According to Chaurasiya and Kumar (2015) analyzes the effect of frequency and diameter on parabolic reflector performance using Matlab simulation software $[6,7]$.

The parabolic reflector antenna is ideal for high gain applications. This efficiency can be improved by moving the feed closer to the reflector, or by increasing the size of the reflector. Parabolic Reflector has better gain and small loss. The fiber-glass parabolic antenna study shows it can be used as a substitute for metallic fiberglass.

\section{EXPERIMENTAL SETUP}

\subsection{Methodology}

The method adopted involves:

- Design of a fiberglass parabolic antenna;

- Antenna installation;

- Testing of the fiberglass parabolic antenna using a handheld microwave device.

\subsection{Parabolic Antenna design}

A parabola is the set of points in the plane that are equidistant from a point (the focus) and a line (the directrix). The general equation of a parabola in terms of its focal length $F$ is (equation (1)) [7]:

$$
y=a \cdot x^{2}
$$

where: $\mathrm{x}$ is radius, a- focal length.

Where $a$, is expressed in terms of the focal length (equation (2)).

$$
a=\frac{1}{4 \cdot F}
$$

2.2.1. Length of a parabolic segment

The formula for calculating the length $\mathrm{L}$ of a parabolic segment from the vertex to the edge is (equation (3)):

$$
L=\frac{\ln \cdot\left(\sqrt{\Omega^{2} \cdot D^{2}+1}+a \cdot D\right)}{4 \cdot \pi}+\frac{D \cdot \sqrt{a^{2} \cdot D^{2}+1}}{4}
$$

\subsubsection{Surface area of a parabolic reflector}

The surface area of a parabolic reflector is calculated with the formula (4):

$$
S=\frac{\pi \cdot\left(a^{2} \cdot D^{2}+1\right)^{\frac{3}{2}-1}}{6 \cdot a^{2}}
$$

A parabola is completely described by two parameters, the diameter $\mathrm{D}$ and the focal length $\mathrm{F}$.

\subsubsection{Focal length of the dish}

The focal length of the dish is the point at which the LNB must be placed to attain a perfect signal. It is the distance between the center of the parabolic reflector and the focal point (equation (6)). The focal point is where all the signals converge. A simple parabola cure is shown in figure 1 with diameter $=300 \mathrm{~cm}$ and radius $=150 \mathrm{~cm}$.

Substituting equation (2) into equation (1) gives [7]:

$$
y=\frac{x^{2}}{4 F}
$$




$$
\text { Focallength }(F)=\text { Diameter } \times \frac{F}{D}
$$

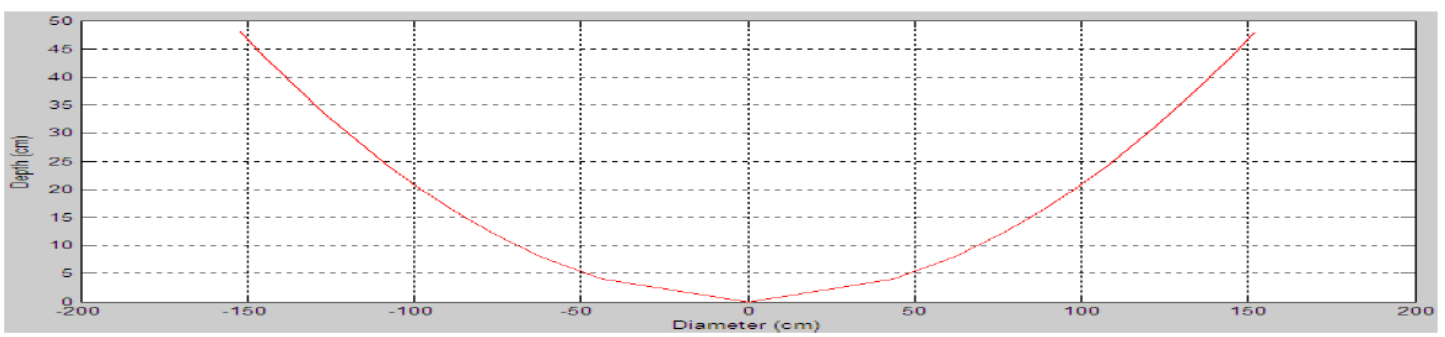

Fig. 1. Graph of a parabola curve.

Ratio F/D usually ranges between 0.3 and 1.0 .

If the efficiency is of the range between 0.5- 0.7 and wavelength is calculated by the expression (7):

$$
\lambda=\frac{e}{f}
$$

Where $\mathrm{c}$ is the speed of light and $\mathrm{f}$ is the frequency of the downlink signal.

$c=3 \times 10^{8}[\mathrm{~m} / \mathrm{s}]$ and $f=11.3 \times 10^{9}[\mathrm{~Hz}]$

\subsubsection{Dish antenna beam width}

The beam width of the satellite dish is inversely proportional to the dish size and operating frequency (equation (8)) [7].

$$
B W=\frac{70 \cdot \pi}{D}[\text { Degrees }]
$$

where: $\mathrm{f}$ is the frequency of the received signal in hertz $=11.3 \mathrm{GHz}, \mathrm{D}$ - diameter of the dish in meters.

\subsubsection{Parabolic antenna efficiency and gain calculations}

The efficiency can also be expressed as the product of a series of terms (equation (9)):

$$
\varepsilon=\varepsilon_{T} \cdot \varepsilon_{A T} \cdot \varepsilon_{5} \cdot \varepsilon_{0}
$$

This efficiency term is in the order of 0.5-0.7 for a well-designed dish antenna (equation (10)) [5]:

$$
G=10 \log _{10} \varepsilon \cdot \frac{4 \cdot \pi}{\mathrm{x}^{2}}=10 \log _{10} \varepsilon \cdot \frac{\left(\pi \cdot D^{2}\right)}{\mathrm{x}^{2}}
$$

where: $\mathrm{G}$ are actual gain in terms of the effective aperture in decibels.

\subsection{Height of installation}

The fiberglass dish can be installed at any height above 6 feet. The already mounted Parabolic antenna must be aligned to point toward the satellite. The angle at which the antenna is eventually situated will vary according to which satellite is selected and at what latitude the antenna is located.

\subsubsection{Antenna installation co-ordinates}

Since the satellite being tracked is the NileSat which is on the longitude $7^{\circ} \mathrm{W}$, the designed and fabricated parabolic reflector antenna dish aiming parameters are thus evaluated. The parameters are required to point an earth station dish antenna to a desired geostationary satellite which is the NileSat $7^{\circ} \mathrm{W}$.

\subsubsection{Azimuth angle}

This is the amount of horizontal rotation in degrees of a dish concerning the true north to point it to the desired satellite (equation (11)). 


$$
(180 \times k 1)+\left((k 2) \times\left(\tan ^{-1}\left(\frac{\tan (\text { longdiff })}{\sin (\text { slat })}\right)\right)\right)
$$

where: $\mathrm{k} 1=0$, for earth station in southern hemisphere, $\mathrm{k} 1=1$, for earth stations in the northern hemisphere, $\mathrm{k} 2=$ -1 .

\subsubsection{Elevation angle}

This is the amount of vertical rotation in degrees a dish makes with the reference horizontal plane (equation (12)) [8].

$$
\text { Elevation }=\tan ^{-1}\left(\frac{\cos [F]-0.1512}{\sin (F)}\right)
$$

where: $F=\cos ^{-1}(\cos ($ longdiff $) \times \cos ($ eslat $))$, Long diff =satlong - eslong, Satlong= satellite longitude, Eslong $=$ earth station longitude, Eslat $=$ earth station latitude.

\subsubsection{Declination angle}

Polar mount dish used for tracking all satellite in the Clarke belt by adjusting the elevation angle (equation (13)).

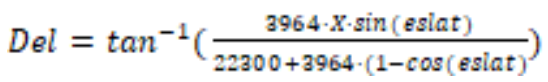

The earth stationed dish is located at the geographic position $\left(3.2^{\circ} \mathrm{E} \quad 6.81^{\circ} \mathrm{N}\right)$.

\section{RESULTS AND DISCUSSION}

The designed antenna using equations 2, 10 and 11 have antenna gain of $49.15 \mathrm{~dB}, 0.0265 \mathrm{~m}$ wavelength and a beam-width of 0.619 degrees. The parabolic reflector antenna was positioned to track the Nile satellite with the dish having an elevation angle of 75.5 degrees, the azimuth angle of 56.16 degrees and declination angle of 1.21 degrees. The surface area and length of the parabolic reflector are $15.94 \mathrm{~m}^{2}$ and $1.5926 \mathrm{~m}$. The simulated radiation pattern of the parabolic antenna is shown in Figure 2 below.

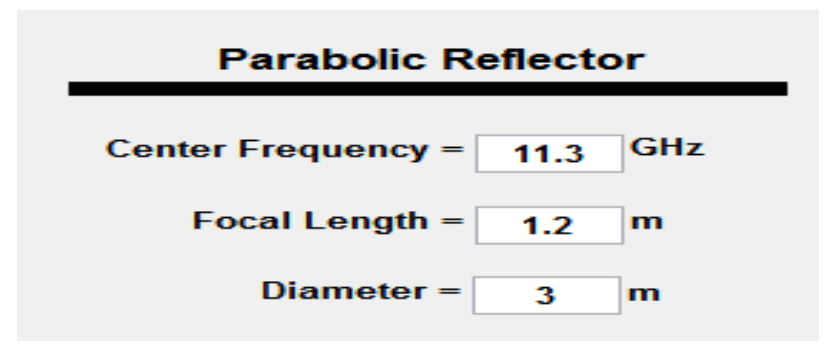

Fig. 2. Parabolic reflector parameters.

The center frequency of $11.3 \mathrm{GHz}$ with the focal length of $1.2 \mathrm{~m}$ and diameter of $3 \mathrm{~m}$ is shown in the electric field pattern and magnetic field pattern of Figure 2. The antenna is described using a spherical coordinate system.

A Radiation pattern is made up of the main beam and side lobes. The E-plane normalized radiation pattern shows the direction of the electric field which is shown in Figure 3. The direction of the electric field determines the polarization of the antenna. 


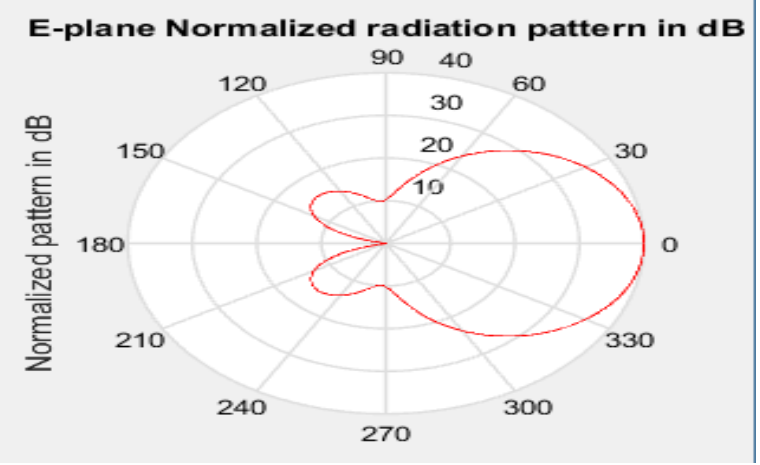

Fig. 3. E-Plane normalized radiation pattern.

Figure 4 shows the H-plane normalized radiation pattern indicating the direction of the magnetic field of the electromagnetic wave. The magnetic field direction is perpendicular to the direction of the electric field.

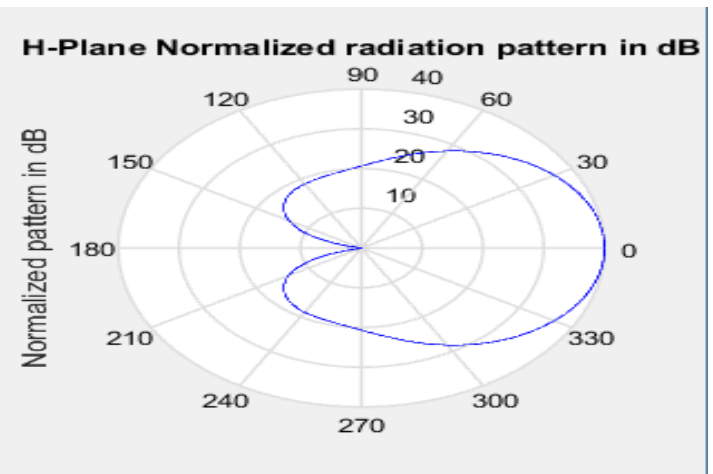

Fig. 4. H-plane normalized radiation pattern.

The radiation pattern beam as shown in figure 5 is pointed out. A pointed pencil beam is required in a radar communication system [7].

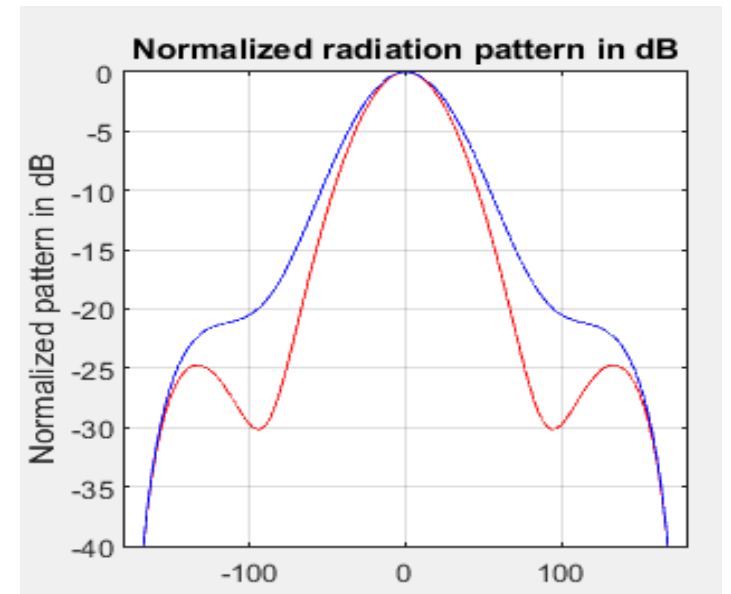

Fig. 5. Normalized radiation pattern of the E- and H- Plane.

Equation (14) and (15) is used for iron and aluminum (Table 1).

Table 1. Aluminum covering

\begin{tabular}{|c|c|c|}
\hline Frequency $(\mathrm{GHz})$ & Penetration depth $(\delta) 10^{-6} \mathrm{~m}$ & $\begin{array}{c}\text { Attenuation coefficient }(\alpha) \\
10^{5} \mathrm{~Np}\end{array}$ \\
\hline 0.500 & 3.553 & 2.814 \\
\hline 0.600 & 3.243 & 3.083 \\
\hline 0.700 & 3.003 & 3.330 \\
\hline
\end{tabular}




\begin{tabular}{|c|c|c|}
\hline 0.800 & 2.808 & 3.560 \\
\hline 0.900 & 2.648 & 3.776 \\
\hline 1.000 & 2.512 & 3.980 \\
\hline 1.200 & 2.293 & 4.360 \\
\hline 1.400 & 2.123 & 4.710 \\
\hline 1.600 & 1.986 & 5.035 \\
\hline 1.800 & 1.872 & 5.340 \\
\hline 2.000 & 1.776 & 5.629 \\
\hline 2.200 & 1.693 & 5.904 \\
\hline 2.400 & 1.621 & 6.167 \\
\hline 2.500 & 1.588 & 6.294 \\
\hline 2.600 & 1.558 & 6.418 \\
\hline 2.700 & 1.528 & 6.541 \\
\hline 10.000 & 0.794 & 12.588 \\
\hline 11.000 & 0.757 & 13.202 \\
\hline 11.500 & 0.740 & 13.499 \\
\hline 12.000 & 0.725 & 13.789 \\
\hline
\end{tabular}

$$
\delta=\frac{1}{\sqrt{\pi \cdot f \cdot[\cdot \sigma \cdot \sigma}}
$$

where: $\delta$ are penetration depth.

$$
\alpha=\frac{1}{8}
$$

where: $\alpha$ are attenuation coefficient.

Here aluminum is assumed to have a conductivity of $3.82 \times 10^{7} \mathrm{~s} / \mathrm{m}$ and relative permeability of one. Equation (16) and (17) used to determine the attenuation coefficient and penetration depth of glass and fiberglass material are:

$$
\begin{gathered}
\alpha=\omega \cdot \sqrt{\frac{\mu \cdot \varepsilon_{0} \cdot \varepsilon_{T}}{2}} \cdot \sqrt{\sqrt{1+\frac{\varepsilon^{11}}{\varepsilon^{1}}}-1} \\
\delta=\frac{1}{\alpha}
\end{gathered}
$$

Relative permittivity for glass is taken to be 6 and the loss tangent to be 0.002 .

The relative permittivity of fiberglass (Table 2) is 3 and the loss tangent is 0.0089 .

Relative permeability of iron (Table 3 ) is 4000 and conductivity is $1.03 \times 10^{7} \mathrm{~s} / \mathrm{m}$.

For the determination of the signal quality received by the designed 3-m fiberglass based dish, a handheld microwave detector (HD Satellite Finder) was used for the test, hence the results were recorded. The test was carried out at 8:00 am $2 \mathrm{pm}$ and $8 \mathrm{pm}$ at $6 \mathrm{hrs}$. interval.

From Table 2 above, the penetration depth of fiberglass reduces as the frequency increases and at $2.7 \mathrm{GHz}$, the penetration depth is $2.294 \mathrm{~m}$. For the fiberglass to be used to construct a parabolic antenna, aluminum or iron or any other conductor is needed to coat the outer covering of the fiberglass as electromagnetic wave do not penetrate deeply the aluminum compared to the fibre-glass. Hence, the reflectivity property of the parabolic antenna is enhanced when coated with aluminum or iron. Since fiberglass is cheaper, constructing a parabolic antenna dish entirely with aluminum or iron will not be economical.

Table 2. Fiberglass composite material.

\begin{tabular}{|c|c|c|}
\hline $\begin{array}{c}\text { Frequency } \\
(\mathrm{GHz})\end{array}$ & $\begin{array}{c}\text { Penetration depth } \\
(\delta) \mathrm{m}\end{array}$ & $\begin{array}{c}\text { Attenuation coefficient } \\
(\alpha) \mathrm{Np}\end{array}$ \\
\hline 0.500 & 12.387 & 0.081 \\
\hline
\end{tabular}




\begin{tabular}{|c|c|c|}
\hline 0.600 & 10.323 & 0.097 \\
\hline 0.700 & 8.848 & 0.113 \\
\hline 0.800 & 7.742 & 0.129 \\
\hline 0.900 & 6.882 & 0.145 \\
\hline 1.000 & 6.193 & 0.161 \\
\hline 1.200 & 5.161 & 0.193 \\
\hline 1.400 & 4.424 & 0.226 \\
\hline 1.600 & 3.871 & 0.258 \\
\hline 1.800 & 3.441 & 0.290 \\
\hline 2.000 & 3.096 & 0.322 \\
\hline 2.200 & 2.815 & 0.355 \\
\hline 2.400 & 2.580 & 0.387 \\
\hline 2.500 & 2.477 & 0.403 \\
\hline 2.600 & 2.382 & 0.420 \\
\hline 2.700 & 2.294 & 0.435 \\
\hline 10.000 & 0.619 & 1.614 \\
\hline 11.000 & 0.563 & 1.775 \\
\hline 11.500 & 0.538 & 1.856 \\
\hline 12.000 & 0.516 & 1.937 \\
\hline
\end{tabular}

This research study shows that fiberglass coated with a film of aluminum or iron or any other conductor is more cost-effective compared with the ones built entirely with metals. Also, Figures 6, 7, 8 shows a signal strength of about $60 \%$ between $11 \mathrm{GHz}$ to $11.5 \mathrm{GHz}$. The signal strength of $60 \%$ and above gave smooth, clean and clear pictures without freeze or crack hence regarded as a strong signal. Signal strength around $50 \%$ may suffer possible cracks/freeze hence regarded as a moderate signal.

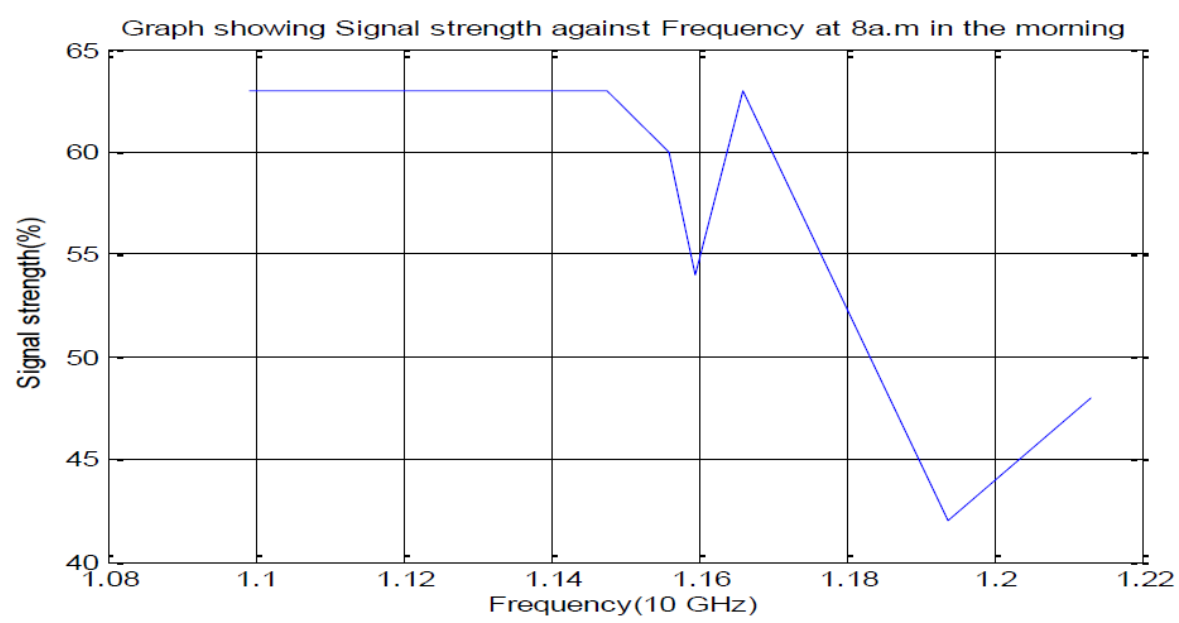

Fig. 6. Plot of signal strength against frequency at $8.00 \mathrm{am}$.

Table 3. Iron.

\begin{tabular}{|c|c|c|}
\hline $\begin{array}{c}\text { Frequency } \\
(\mathrm{GHz})\end{array}$ & $\begin{array}{c}\text { Penetration depth } \\
(\delta) \\
\left(10^{-8}\right) \mathrm{m}\end{array}$ & $\begin{array}{c}\text { Attenuation coefficient } \\
(\alpha)\left(10^{6} \mathrm{~Np}\right)\end{array}$ \\
\hline 0.500 & 11.087 & 9.019 \\
\hline 0.600 & 10.121 & 9.880 \\
\hline 0.700 & 9.371 & 10.671 \\
\hline 0.800 & 8.765 & 11.408 \\
\hline 0.900 & 8.264 & 12.100 \\
\hline 1.000 & 7.840 & 12.755 \\
\hline 1.200 & 7.156 & 13.972 \\
\hline 1.400 & 6.625 & 15.092 \\
\hline 1.600 & 6.198 & 16.134 \\
\hline 1.800 & 5.843 & 17.112 \\
\hline
\end{tabular}




\begin{tabular}{|l|l|l|}
\hline 2.000 & 5.543 & 18.038 \\
\hline 2.200 & 5.285 & 18.918 \\
\hline 2.400 & 5.060 & 19.760 \\
\hline 2.500 & 4.958 & 20.167 \\
\hline 2.600 & 4.862 & 20.567 \\
\hline 2.700 & 4.771 & 20.958 \\
\hline 10.000 & 2.479 & 40.335 \\
\hline 11.000 & 2.363 & 42.303 \\
\hline 11.500 & 2.311 & 43.254 \\
\hline 12.000 & 2.263 & 44.185 \\
\hline
\end{tabular}

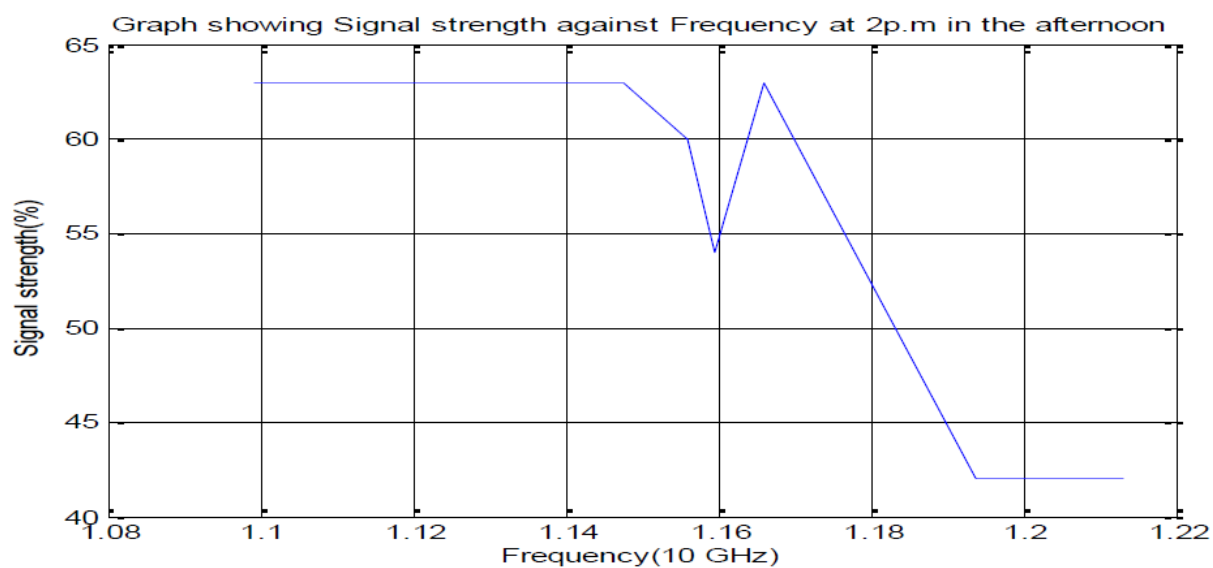

Fig. 7. Plot of Signal strength against Frequency at $2 \mathrm{pm}$.

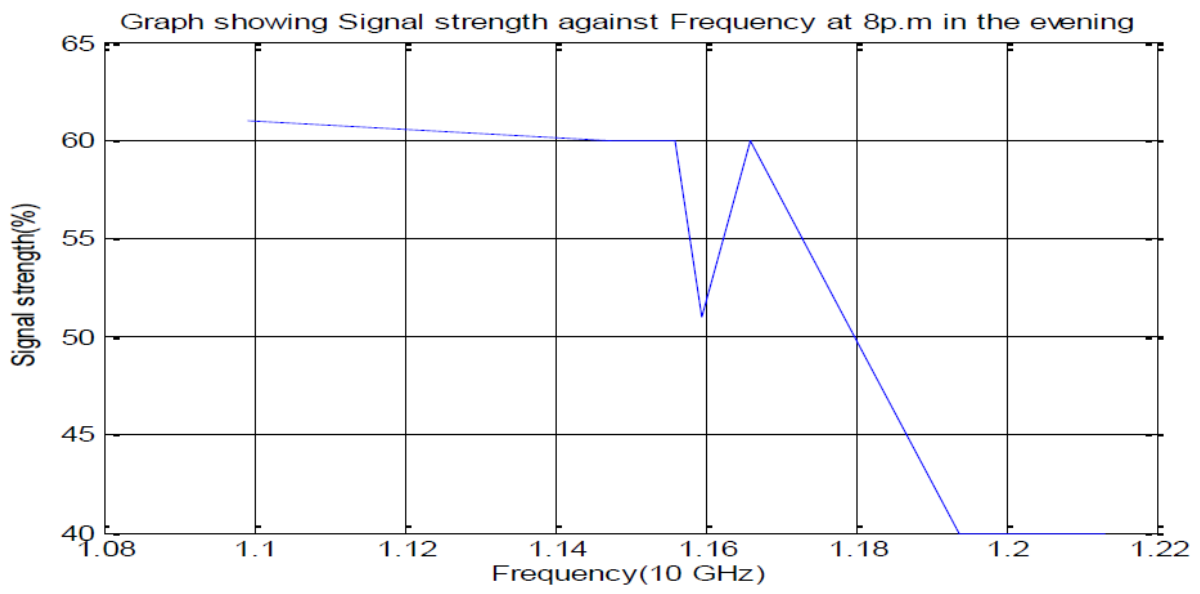

Fig. 8. Plot of Signal strength against Frequency at $8 \mathrm{pm}$.

\section{CONCLUSIONS}

The result shows the locally designed fiberglass composite parabolic antenna showing the E-plane and H-plane radiation pattern. The parabolic antenna is used to receive a television signal at $11.3 \mathrm{GHz}$ with the signal strength of $60 \%$. This study shows that fiberglass is a viable alternative material for designing a parabolic antenna. Due to the ease of production, it can conveniently replace the conventional metallic parabolic antenna.

To ensure the efficient usage of limited financial resources in developing countries, the development of this work is strongly recommended because of the following reasons: Firstly, the coating of the outer covering of the designed fiberglass with aluminum or iron allows an enhanced reflectivity property of the parabolic antenna thus allowing light which enters it travelling parallel to the axis of the symmetry to be reflected towards the focus.

Secondly, since fiberglass is cheaper, constructing a parabolic antenna dish entirely with aluminum or iron may not be economical. Accordingly, results obtained in this work shows that fiberglass coated with a film of 
aluminum or iron or any other conductor is more cost-effective compared with the ones built entirely with metals.

Finally, experimental results show that fiberglass parabolic antenna gives a signal strength of about $60 \%$ between $11 \mathrm{GHz}$ to $11.5 \mathrm{GHz}$. Accordingly, the $60 \%$ signal strength is characterized as having a smooth, clean and clear pictures without freeze or cracks hence, the signal is very strong.

\section{REFERENCES}

[1] www.archive.mu.ac.in>myweb-test (12.09.2019).

[2] Morozov, S., Gaio, M., Maier, S.A., Supienza, R., A metal-dielectric parabolic antenna to direct single photons, Physics. Optics, 2018, p. 1-11.

[3] Janhunen, P., Olsson, A., Karlsson, R., Griebmeier, J.M., Motivation and possibilities of affordable lowfrequency radio interferometry in space, Astronomy and Astrophysics, 2003, p. 1-7.

[4] Ullah, M.H, Islam, M.T., Ahsan, M.R., Mahad, W.N., Lateef, T.A., Uddin, M.J.,). A low-cost fiberglass polymer resin dielectric material based microstrip patch antenna for multiband applications., Science and Engineering of Composite Materials, vol. 23, no. 4, 2015, p. 447-452.

[5] Papageorgiou, I., Investigation and design of high gain, low sidelobes, compact antennas at E-band, Master thesis in Antennas, Chalmers University of Technology, Gothenburg, Sweden, 2012.

[6] Sharp, G.R, Wanhainen, J.S., Ketelsen, D.A., A Fabrication method for precision antenna reflectors for space flight and ground test, NASA Technical Paper, 1991.

[7] Chaurasiya, K., Kumar, S., Design and analysis of parabolic reflector using Matlab, International Journal of Advanced Research in Electrical, Electronics and Instrumentation Engineering, vol. 4, no. 3, 2015, p. 1367-1373. [8] Roddy, D., Coolen, J., Electronic communications, Pearson Education, 2007. 